\title{
The mechanics of setting up a COVID-19 response: Experiences of the COVID-19 epidemic from Groote Schuur Hospital, Cape Town, South Africa
}

\begin{abstract}
M Mendelson, ${ }^{1,2} \mathrm{MBBS}, \mathrm{PhD} ; \mathrm{L}$ Booyens, ${ }^{3} \mathrm{MB} \mathrm{ChB} ;$ A Boutall, ${ }^{4} \mathrm{MB} \mathrm{ChB}, \mathrm{FCS}$ (SA), Cert Gastroenterology (Surgery); L Cairncross, ${ }^{4}$ MB ChB, FCS (SA), MMed (Surgery); G Calligaro, ${ }^{2,5}$ BSc Hons, MB BCh, Dip PEC (SA), MMed (Medicine), FCP (SA), Cert Pulmonology (SA); J A Dave, ${ }^{2,6} \mathrm{MB}$ ChB, PhD, FCP (SA), Cert Endocrinology and Metabolism (SA); FRCP (Lond); S Dlamini, ${ }^{1,2}$ MB ChB, FCP (SA), Cert ID (SA) Phys; S Dyer, ${ }^{7}$ MB ChB, FCOG, MMed (Obstetrics \& Gynaecology), PhD; B Eick, ${ }^{3} \mathrm{MB} \mathrm{ChB}, \mathrm{MD}$; K Fieggen, ${ }^{2,8} \mathrm{MB}$ ChB, FC Paed (SA), Cert Medical Genetics (SA); P Frankenfeld, ${ }^{2} \mathrm{MB}$ ChB, FCP (SA); J Hoare, ${ }^{9} \mathrm{MB}$ ChB, DMH, MRCPsych (UK), FC Psych (SA), MPhil (Neuropsychiatry), PhD; R Hofmeyr ${ }^{10}$ MB ChB, Dip PEC (SA), DA, MMed (Anaesthesia), FCA, FAWM; J Joska, ${ }^{9}$ MB ChB, MMed (Psych), PhD, FC Psych (SA); I Joubert, ${ }^{11}$ MB BCh, DA (SA), FCA (SA) (Critical Care); R Krause, ${ }^{12} \mathrm{MB}$ ChB, MFamMed, MPhil (Palliative Medicine), PD Health Professional Education; A Kropman, ${ }^{13} \mathrm{MB}$ ChB, Dip PEC (SA), FCEM; D Levin, ${ }^{2,14} \mathrm{MB}$ BCh, MBA, FCP (SA), Cert Gastroenterology (SA);

D Maughan, ${ }^{2} \mathrm{MB}$ ChB, MMed (Medicine), FCP (SA); G Meintjes, ${ }^{1,2} \mathrm{MB}$ ChB, FRCP (UK), FCP (SA), MPH, PhD; E Muller; ${ }^{4} \mathrm{MB}$ ChB, PhD; N Ntusi, ${ }^{2}$ DPhil, MD; N Papavarnavas, ${ }^{2}$ MB ChB, Dip HIV Man (SA), DTM\&H, Dip Int Med (SA), MMed (Medicine), FCP (SA); B Patel, ${ }^{3}$ BSc, MB ChB, MFamMed, FCFP, FCPHM, BSc (Med) (Bioethics and Health Law); J Peter, ${ }^{2,15}$ MB ChB, PhD; P Raubenheimer, ${ }^{2} \mathrm{MB}$ BCh, FCP (SA); Q Said-Hartley, ${ }^{16} \mathrm{MB}$ ChB, FC Rad Diag (SA); P Singh, ${ }^{2} \mathrm{MB}$ ChB, FCP (SA), MMed (Medicine), Dip HIV Man (SA); $S$ Wasserman, ${ }^{1,2}$ MB ChB, MMed (Medicine), Cert ID (SA) Phys; on behalf of the Groote Schuur Hospital COVID-19 Response Team*
\end{abstract}

\author{
${ }^{1}$ Division of Infectious Diseases and HIV Medicine, Faculty of Health Sciences, University of Cape Town, South Africa \\ ${ }^{2}$ Department of Medicine, Faculty of Health Sciences, University of Cape Town, South Africa \\ ${ }^{3}$ Groote Schuur Hospital, Western Cape Department of Health, South Africa \\ ${ }^{4}$ Division of General Surgery, Faculty of Health Sciences, University of Cape Town, South Africa \\ ${ }^{5}$ Division of Pulmonology, Groote Schuur Hospital, Western Cape Province Department of Health, South Africa \\ ${ }^{6}$ Division of Endocrinology, Faculty of Health Sciences, University of Cape Town, South Africa \\ ${ }^{7}$ Department of Obstetrics and Gynaecology, Faculty of Health Sciences, University of Cape Town, South Africa \\ ${ }^{8}$ Division of Human Genetics, Faculty of Health Sciences, University of Cape Town, South Africa \\ ${ }^{9}$ Department of Psychiatry, Faculty of Health Sciences, University of Cape Town, South Africa \\ ${ }^{10}$ Department of Anaesthesia and Perioperative Medicine, Faculty of Health Sciences, University of Cape Town, South Africa \\ ${ }^{11}$ Division of Critical Care Medicine, Faculty of Health Sciences, University of Cape Town, South Africa \\ ${ }^{12}$ School of Public Health and Family Medicine, Faculty of Health Sciences, University of Cape Town, South Africa \\ ${ }^{13}$ Division of Emergency Medicine, Faculty of Health Sciences, University of Cape Town, South Africa \\ ${ }^{14}$ Division of Gastroenterology, Faculty of Health Sciences, University of Cape Town, South Africa \\ ${ }^{15}$ Division of Allergy and Clinical Immunology, Faculty of Health Sciences, University of Cape Town, South Africa \\ ${ }^{16}$ Department of Radiology, Faculty of Health Sciences, University of Cape Town, South Africa \\ ${ }^{*}$ Groote Schuur Hospital COVID-19 Response Team - see supplementary file for full list (http://www.samj.org.za/public/sup/15157.pdf)
}

Corresponding author: M Mendelson (marc.mendelson@uct.ac.za)

\begin{abstract}
The SARS-CoV-2 pandemic has challenged the provision of healthcare in ways that are unprecedented in our lifetime. Planning for the sheer numbers expected during the surge has required public hospitals to de-escalate all non-essential clinical services to focus on COVID19. Western Cape Province was the initial epicentre of the COVID-19 epidemic in South Africa (SA), and the Cape Town metro was its hardest-hit geographical region. We describe how we constructed our COVID-19 hospital-wide clinical service at Groote Schuur Hospital, the University of Cape Town's tertiary-level teaching hospital. By describing the barriers and enablers, we hope to provide guidance rather than a blueprint for hospitals elsewhere in SA and in low-resource countries that face similar challenges now or during subsequent waves.
\end{abstract}

S Afr Med J 2020;110(10):968-972. https://doi.org/10.7196/SAMJ.2020.v110i10.15215

As of 1 September 2020, South Africa (SA) had recorded 625056 confirmed COVID-19 cases, 538604 recoveries, 72424 active cases and 14088 deaths. $^{[1]}$ Just five and a half months earlier at Groote Schuur Hospital (GSH), the academic teaching hospital of the University of Cape Town (UCT), a 19-year-old SA student who had returned from Europe became the first person under investigation (PUI) at our hospital to be tested for SARS-CoV-2, the novel coronavirus causing the global respiratory pandemic. In the short time since then, 3487 PUIs have been admitted and 1781 confirmed cases of COVID-19 among inpatients have been identified (Western Cape Provincial Health Data Centre, unpublished), the number of dedicated COVID-19 beds has grown from 1 to over 250 at its peak, and the number of doctors working full-time in the COVID-19 service expanded from 5 to 114 . Although the figures 
and the speed of change are impressive, the journey to this point comprises an equal number of failures and successes from which we have learned and which we now share to provide guidance to others faced with similar challenges. In this, the first of two articles, we describe how to optimise the hospital's response, while details of clinical management are dealt with in the second article (https://doi. org/10.7196/SAMJ.2020.v110i10.15157).

\section{Developing a whole-of-hospital staffing model}

The relentless but incremental nature of the increase in patient numbers found us in an iterative process to develop our staffing model. Ward-based teams, which contributed members to separate acute intake teams in a cycling roster, ensured continuity of care on the wards and enough 'bodies on the ground' to cope with the increasing number of daily admissions. However, it soon became clear from the sheer workload that this model required a 'wholeof-hospital' approach, drawing staff from outside the Department of Medicine across all departments, many of which do not usually involve themselves with acute medical admissions.

Our ward-based teams started with 16 doctors on 2 wards, expanding to 95 doctors from all specialties, distributed in teams across 11 wards. Six doctors per 8-hour shift formed the intake teams, covering admissions 24/7. The intake teams were divided in two groups: physicians (who worked in a 5-day rolling cycle, 3 days on, 2 off, with 12 teams in all) as well as a large group of doctors from specialties outside medicine who worked in an 8-hour shift system alongside their medical colleagues. This system allowed for optimal service provision and adequate cover for staff illness, as well as for ward cover after hours.

Staffing pressures were heightened in the early weeks of our response by the inexplicable need to open new wards on a Friday afternoon, which meant reallocating staff from non-COVID-19 teams at the start of the weekend, without adequate training in infection prevention and control (IPC). With time we became better at predicting the trend in numbers and were aided by introduction of planned staffing allocations. Early planning, training of staff, and weekday openings before the pressures become too great are strongly recommended.

Our modus operandi progressively evolved from the traditional hierarchical structure to a flattened structure with the team-based approach. Teams working on intakes became multidisciplinary with different levels of experience (see 'The power of teams'). Departments allocated staff to the COVID-19 service in such a way that most other critical services in the hospital have been able to continue. However, many challenges were faced in transforming traditional disciplinefocused, priority-setting activities to one focused predominantly (but not exclusively) on a single focus, COVID-19. Long and numerous discussions to reach consensus were needed to face the reality that many services had to be compromised. Which emergency services should remain and which non-emergency services can be temporarily withdrawn are contentious, and the definition of 'emergency' can be debated. The incremental nature of the scale-up necessitated that each step of the process be revisited, repeatedly, discussing how to balance priorities, and what could be preserved while getting prepared to meet the growing demands of COVID- 19 . Early engagement to reach consensus on progressive redeployment of staff is highly recommended.

Staff infections with SARS-CoV-2 have been high, negatively affecting morale and reducing the size of teams, thereby adding extra workload to colleagues who remain on duty. Exhaustion and related illness were common prior to reaching the staff numbers attained through the whole-of-hospital approach. To protect the health workforce from burnout and sickness, we strongly urge all institutions to instigate such a structure at the earliest possible opportunity.

\section{The power of teams}

As COVID-19 is first and foremost a respiratory infection, the clinical burden of care is usually taken up by physicians, who lead the service. Two doctors (including senior and junior grades) from departments other than medicine joined in each ward and admission team shift. The creation of multidisciplinary intake and ward teams helped to overcome fear of the illness, bridge knowledge gaps, and equip and support doctors who felt overwhelmed and out of their comfort zone. Whenever possible, we introduced non-physician members to the ward teams before allocation to frontline intake teams, helping to build confidence. Consultants working shifts in the Emergency Unit (EU) alongside middle and junior doctors engendered buy-in across the board, and limited resentment related to increased workloads. It created a unique transversal camaraderie under challenging circumstances.

We advise that non-physicians should be paired with an anchor senior medical consultant and medical middle-grade doctor/s. Whenever possible, these teams should remain constant. This flat structure allows for rapid knowledge accumulation for nonphysicians, and, at its core, a supportive environment focused on a common mission. We undertook a short web-based meeting daily at $08 \mathrm{~h} 00$ to check on all parts of the service. Similarly, clinical meetings in person, limited in size and with appropriate IPC precautions, provided the various disciplines with a sense of unity and enabled voices to be heard and rapid learning to be shared.

By keeping teams together, institutional process and clinical memory are entrenched, thereby improving service delivery and efficiency. We found that teams respond to adversity with mutual support, and when one member goes off sick, others rally round to fill the gap.

\section{Planning PUI and COVID-19 wards}

Ideally, the commissioning of PUI and COVID-19 wards should be a planned and formal process with the participation of multiple stakeholders. The speed at which the epidemic hits is difficult to contend with, despite warnings from other countries, as is early oscillation in case numbers, which can lead to a false sense of security. Contending with the collapsing of non-emergency services added complexity to the decision-making process. From our experiences, we strongly recommend that when it comes to planning new wards, hospitals work on the basis of being at least 2 weeks ahead of the curve.

Important stakeholders for planning include nursing management, IPC staff, information technology, engineering, and catering and cleaning services. Predicting how many PUI and COVID-19 wards will be required is difficult and is largely dependent on local epidemiology of SARS-CoV-2 in a given region. As a rule, plan for double the number of COVID-19 wards than PUI wards at the peak of the surge. We started with one ward (with isolation rooms) for both PUI and COVID-19 patients and have evolved to having 4 PUI and 7 COVID-19 wards, with additional PUI and COVID19 wards in obstetrics, psychiatry and intensive care contributing further to the overall hospital's response. PUI wards for mental healthcare users present particular problems owing to mobility of patients admitted without a respiratory component to COVID-19. In this regard, smaller spaces are essential, as is prioritisation of testing to give a rapid result. Indeed, our greatest challenge happened 
when the laboratory turnaround time for the SARS-CoV-2 test increased to up to 5 days, at which point a bottleneck occurred in the PUI wards. Ensuring prioritisation of inpatient tests is critical (see article 2).

Patient flow into and out of the ward must be well planned. We suggest that staff and patient entry is through different entrances, with adequate, clearly signed areas for donning and doffing of personal protective equipment (PPE). Each ward should have changing rooms close by to avoid movement around the hospital in PPE. Wards with individual rooms are ideal for housing PUIs, but in open wards, consider making 'virtual cubicles' with floor tape to demarcate a contamination space around the bed. The demarcation should be a minimum of 1 $1.5 \mathrm{~m}$ from the bed in either direction, which serves as a visible reminder relating to IPC, and PPE stations should be set up at highly visible points in each ward for changing of gloves, aprons, etc.

Where equipment is concerned, monitors, particularly those measuring oxygen saturation, are often at a premium. One per bed is recommended in high-care areas. Negative pressure ventilation single rooms are optimal for nursing patients co-infected with SARS-CoV-2 and Mycobacterium tuberculosis. Other infrastructure considerations include ensuring correct numbers and functioning of oxygen points in the wards. If planning to use high-flow nasal cannula (HFNC) oxygen in the wards as opposed to the intensive care unit (ICU) setting, it is important to ensure that the hospital oxygen reticulation system will support it. Basic supplies of stethoscopes, syringe drivers, a resuscitation trolley, cardiac monitors, infusion pumps, and an adequately stocked medicine trolley are essentials.

\section{Expanding ICU capacity}

We identified all spaces in the hospital that could feasibly house ventilated patients and their equipment and IPC needs. A new structure of ICU firms was created, bolstered by additional staff from other departments, such that each firm would manage 12 18 patients, and a staffing plan (medical, nursing, allied and administrative staff) based on a shift system was developed for existing and new COVID-19 beds. We trained staff in PPE in all new areas to enable practice and understanding of the needs for their daily work. The provincial triage policy for COVID-19 (https://www. westerncape.gov.za/assets/departments/ health/h83_2020_covid-19_covid_critical_ care_triage_and_decision_tool.pdf) was adopted and widely disseminated within the province.

Pivotal to our success has been a strong commitment from hospital and provincial management and development of a strong provincial critical care plan enabling all hospitals on the platform to assist in contributing to the care of critically ill COVID-19 patients. Rapid and appropriate procurement of equipment, medications, disposable devices and PPE through wellmanaged supply chains was a critical enabler, as was the hospital's commitment to increase staff working in critical care almost 3.5-fold.

Forward planning is key. The final increase of 44 additional ICU beds for ventilating COVID-19 patients required appropriation of areas and commissioning of new ICU beds, such that 2 days' worth of projected admissions would be available at any point in time. This was only possible by accepting that nurse-to-patient ratios would have to be reduced to some extent to allow for all the additional beds.

\section{Setting up specific \\ clinical areas}

\section{Organising the testing centre}

The only certainty in the evolution of our hospital's testing centre (TC) has been constant change, posing considerable challenges: a new space; a new screening form, reporting process or case/testing definition; rotating staff often unfamiliar with infection control and testing techniques; and new testing methods.

Physical space is a critical determinant of safety in managing potentially infectious patients, and as numbers for testing increased from 50 to 200 per day between March and June, our service moved from a single cubicle in a general ward, to a repurposed transport office with swabbing outdoors, to the final stand-alone newly built TC to meet the surge (Fig. 1).

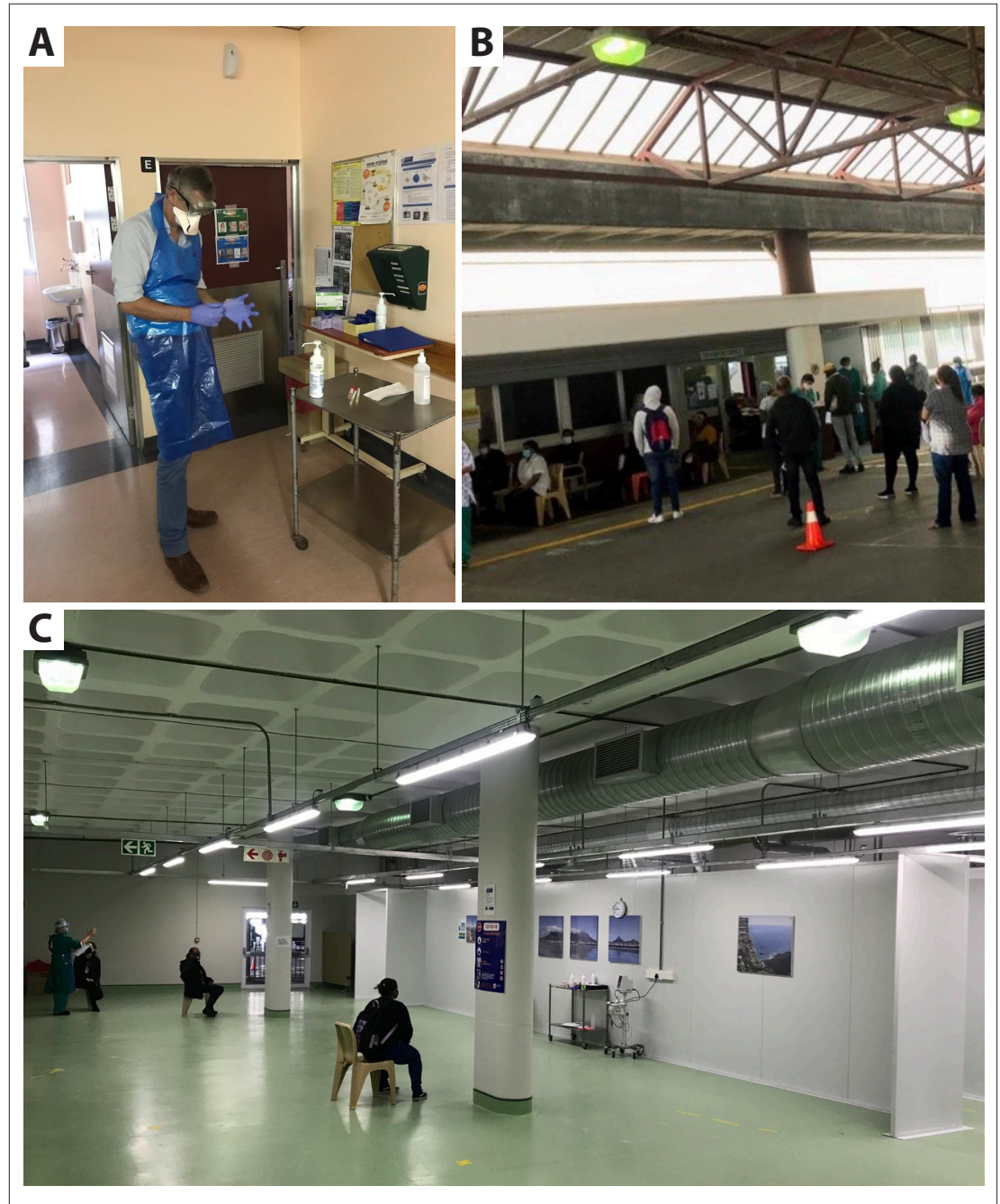

Fig. 1. Evolution of the Groote Schuur Hospital Testing Centre from a single room on a single ward (A), to a repurposed transit lounge with outside swabbing (B), to the final stand-alone product (C). 
We allocated a senior medical manager to oversee the process, development and enactment of standard operating procedures, and ensure compliance with IPC practices. TC needs were included as daily agenda items for hospital management. Advocacy for staffing on the whole-of-hospital basis saw many departments rostering members to do shifts in the TC, and we made use of provincial and student volunteer groups as the surge played out.

A provincial change in testing strategy that occurred towards our peak resulted in reduced numbers being tested. Coupled with a change from nasopharyngeal to mid-nasal turbinate sample collection that the client can perform themselves, fewer experienced clinicians have been needed. They could be re-deployed to ward teams, leaving a small, experienced group of clinicians and nursing and support staff.

Planning needs to take account of human resource needs for communicating results to clients (done off-site by volunteers, which frees up time for staff in the centre to focus on the core business of triage, screening, testing and education), ensuring easy flow of patients requiring urgent referral to the emergency unit (we positioned our TC next to its entrance), and as with other areas of the service, staff absenteeism due to COVID-19 or other reasons.

\section{Organising the Emergency Centre}

Historically, our Emergency Centre (EC) comprises a separate EU and Trauma Unit (TU). Our initial plan involved identified PUIs being triaged into a consulting room for further evaluation, before admission to a PUI ward. Four months later, much has changed.

As numbers of admissions increased, we created 'hot' zones in both the EU and the TU, which for the EU was too small to allow staff to keep pace and stabilise patients adequately. A dedicated 5-stretcher bay was immediately full, and the need to move patients rapidly from the EU to wards meant that PUI wards become de facto emergency wards themselves. Expansion to a 12-stretcher bay in the EU failed to relieve the situation, and it was not until we switched to a model combining the EU and TU, so that all 'hot' patients irrespective of their presentation were triaged to the 'hot' side, while trauma and medical patients not fulfilling PUI criteria were triaged to the 'cold' side, that patient flow into the hospital could be managed adequately.

The importance of stabilising patients in a centralised place with facilitated teamwork cannot be overstated. Clear IPC challenges exist in ensuring that 'cold' patients really are 'cold', which required similar IPC practices across both units. To increase space in the units required repurposing many areas such as patient and family waiting rooms, storage areas and patient bathrooms to create triage, consultation, treatment and holding areas, as well as staff donning and doffing rooms.

Despite stockpiling and tightening control of PPE early on, restrictions in access to stock within the unit and an increase in supply were needed to meet the daily needs. Other focuses for IPC included marking the floors of all bays so that stretchers were adequately spaced, and limiting staff numbers allowed in tea rooms and offices. We did not move patients between bays. Surges over the course of each day led to variations in space requirements, so a flexible area that could be used interchangeably from either 'hot' or 'cold' sides was created. To limit transmission further, escorts were prevented from entering the unit, but their contact details were captured to aid communication with family members. We recommend establishing a communication team early to handle family enquiries. Administering deaths created its own challenges. We swabbed all dead bodies. Muslim death certificates were completed at the time of certification of death, and if the clinical history or presentation supported COVID-19, 'COVID-19 suspect' was entered into the death form.
Other death certificates were only completed after results were received for the swabs done at the time of death.

\section{Specific challenges relating to the COVID-19 service}

\section{Supporting referral hospitals in a time of severe} resource constraint

Whatever pressures were experienced at our large hospital, the situation was worse in smaller district and regional referral hospitals, which have less space to expand into, and far fewer staff. That meant continuing to rotate staff as part of the service platform and providing an understanding that most referrals would be accepted. To ensure optimal communication, heads of departments from secondary-level hospitals joined daily management calls.

Having a dedicated COVID-19 phone carried by a senior member of the COVID-19 admission team, 24/7, helps with clinical support, as did moving to an app-based referral system, capable of interactive discussion online. We used Vula (see 'Setting up paperless systems'). As ICU beds and HFNC support were offered predominantly at our hospital, all referrals of critically ill patients came our way. Transfer of unstable patients with high oxygen requirements is very risky, so we recommend early transfer of patients deemed candidates for intensive care, with most intubations taking place at the central hospital. Despite this policy, transfers of these patients often resulted in respiratory deterioration in transit, and it was not uncommon to need to intubate patients immediately on arrival from outside centres. This situation was worsened by delays in transfer of patients from outlying hospitals, due to pressures on the ambulance services. These patients are on a very delicate balance, and any exertion, including moving across stretchers and even change in position on a bed, can cause profound oxygen desaturation. We had many discussions about ethics and resource allocations, but our central role in supporting referral hospitals whenever feasible remained foremost. The last key determinant of success in supporting referral hospitals is the ambulance services, and the regular communication needed with emergency services to understand how best to facilitate transfers. Many transfers could only be completed after hours, which required us to alter staff allocation on the intake teams to ensure adequate cover for the $16 \mathrm{~h} 00$ - $00 \mathrm{~h} 00$ rush of patients.

\section{Setting up paperless systems}

Conventional paper-based medical notes and referral systems provide infection prevention challenges with movement and handling of folders and notes from contaminated areas. We trialed and implemented three paperless platforms to address this problem.

Trello (https://trello.com) is a cloud-based application that allows patient tracking, and display of patient location, history, clinical and laboratory parameters and radiology images. A separate Trello board formed part of our admissions process, allowing analysis of bed statistics per ward and patient bookings in real time.

Vula (https://www.vulamobile.com/) is a secure, applicationbased platform, which we moved to in order to take advantage of its referral interface. A preset drop-down menu enables efficient accrual of patient information and management details. It supports effective triage and more efficient patient care, reducing the need for telephonic consultations, which become onerous during busy intakes.

IDRIS is a bespoke web-based electronic records system developed at UCT, accessible via mobile devices, and currently being trialled in our COVID-19 service. Its advantage is in allowing instant sharing of information with real-time clinical audits, enabling us to track flow 
and trends in the hospital. The interface has the potential to be linked with our laboratory and radiology databases.

One challenge to a paperless system has been that many clinicians wanted to have a printed copy of the patient's notes generated from IDRIS for legal surety and for transfer or discharge purposes. A lack of printers on the wards and in the EU meant that many preferred to continue with the old paper-based system. Technical glitches also occurred, with such relatively small tensions putting paid to aspirations despite the recognised benefits. Workshopping such issues is critical to success.

\section{Supply chain management}

COVID-19 has resulted in a massive increase in hospital spend. Supply chain processes had to be scaled up in order to accommodate service and staff needs, including adequate provision of PPE for all staff, which increased the hospital's spend from ZAR2 million annually to ZAR18 million in just the past 4 months. Manufacturer and supplier constraints together with significant price escalations make this difficult to navigate. Additional consumables such as stethoscopes, blood pressure cuffs, alcohol-based hand rub and thermometers for new wards are needed, as is hardware such as additional ventilators, out-of-ICU oxygen delivery via HFNC, monitors and infusion pumps, requiring an expenditure of ZAR10 - 12 million.

Close communication and co-operation with the provincial supply chain has been critical to success in large projects such as the building of a TC, an additional intensive care ward and two theatres. Strict adherence to the supply chain processes, and leadership, oversight and strict management of the activities from the head of GSH's finance unit, have also been essential. The complexity of managing a changing landscape in the middle of an evolving disease has highlighted the need for strong support services units working in unison to ensure staff and patient safety. These are principles that should not been compromised.

\section{Suimmari y}

Achieving a whole-of-hospital staffing model to prevent burnout and collapse of clinical care, and the formation of multidisciplinary teams, has been a central enabler to what success we have achieved.
Staying ahead of the curve by 2 weeks in planning ward openings is highly recommended, as is dividing emergency centres into 'hot' and 'cold' areas to stabilise the large numbers of COVID-19 patients with hypoxic pneumonia before moving them to the wards. These critical enablers were achieved late in our response, and we urge colleagues elsewhere not to similarly delay planning and implementation.

For most of us, the SARS-CoV-2 pandemic has provided a unique challenge in our medical careers, and the value of learning from shared experiences cannot be understated. Reporting bias will always exclude a frank description of the conflict that erupts when working under tremendous pressure and resource limitations, but by sharing our experiences of what has worked and identifying some of the major challenges, we hope that the path can be made smoother for others as they move towards the surge.

\section{Declaration. None.}

Acknowledgements. We acknowledge with immense gratitude the tremendous contribution to the success of the COVID-19 response at GSH from our nurses, allied medical professionals, and hospital and provincial managers, as well as every last porter, cleaner, caterer and other support staff, and the volunteers who have contributed to our service. We dedicate this article to them, and to all our patients - those who died, and those who have left our hospital to return to friends and family.

Author contributions. MM conceived and co-ordinated the manuscript. All authors wrote parts and/or led the services or administrative tasks described, and all authors edited and approved the final manuscript for submission.

Funding. None.

Conflicts of interest. None.

1. National Institute for Communicable Diseases. COVID-19 surveillance by province (South Africa). https://gis.nicd.ac.za/portal/apps/opsdashboard/index.html\#/0ec12f471aaa4055999366669b38482d (accessed 15 July 2020)

Accepted 12 August 2020 